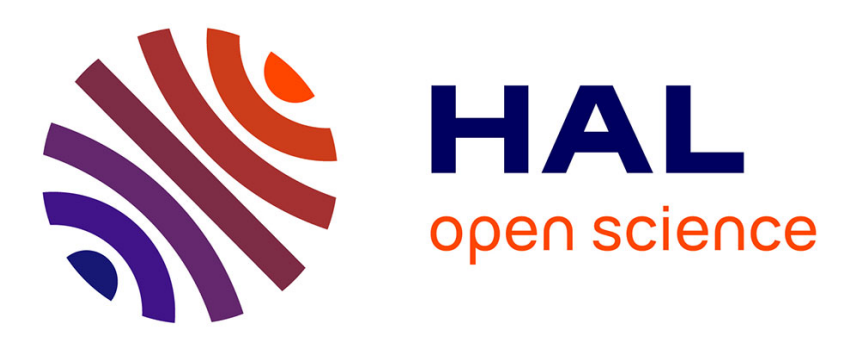

\title{
Aggregation properties of gangliosides: influence of the primary and secondary structure of the headgroup
}

\author{
L. Cantù, M. Corti, D. Acquotti, S. Sonnino
}

\section{To cite this version:}

L. Cantù, M. Corti, D. Acquotti, S. Sonnino. Aggregation properties of gangliosides : influence of the primary and secondary structure of the headgroup. Journal de Physique IV Proceedings, 1993, 03 (C1), pp.C1-57-C1-64. 10.1051/jp4:1993106 . jpa-00251549

HAL Id: jpa-00251549

https://hal.science/jpa-00251549

Submitted on 1 Jan 1993

HAL is a multi-disciplinary open access archive for the deposit and dissemination of scientific research documents, whether they are published or not. The documents may come from teaching and research institutions in France or abroad, or from public or private research centers.
L'archive ouverte pluridisciplinaire HAL, est destinée au dépôt et à la diffusion de documents scientifiques de niveau recherche, publiés ou non, émanant des établissements d'enseignement et de recherche français ou étrangers, des laboratoires publics ou privés. 


\title{
Aggregation properties of gangliosides: influence of the primary and secondary structure of the headgroup
}

\author{
L. CANTÜ, M. CORTI*, D. ACQUOTTI and S. SONNINO
}

Study Center for the Functional Biochemistry of Brain Lipids, Dept. of Chemistry and Biochemistry, Medical School, University of Milan, Milan, Italy

" Dept. of Electronics, University of Pavia, Pavia, Italy

\begin{abstract}
A comparative discussion on the aggregation properties of gangliosides is presented. It is shown that, thanks to the steric packing features of gangliosides, significative considerations can be made on the primary and secondary structure of their headgroup, starting from their cooperative behaviour, supporting NMR direct observations.

On présente une discussion comparative de propriétés d'agrégation dans l'eau des gangliosides. Ces glycolipides sont des tensioactifs dont la tête polaire, constituée de cycles de sucres, est trés volumineuse et hydratée. En accord avec des données RMN, on montre que le propriétés d'agregation des gangliosides sont assez sensibles à la structure primaire et secondaire de leur tête polaire.
\end{abstract}

\section{INTRODUCTION}

In the last years a great deal of work has been done on the physical characterization of ganglioside aggregates by laser light scattering technique [1].

Gangliosides are natural glycosphyngolipids occurring in the plasma membranes of vertebrate cells and are particularly abundant in the nervous system [2]; they are double tailed amphiphilic molecules in which a ceramide lipid portion, constituted mainly by $C 18$ and $C 20$ sphingosine and $\mathrm{C} 18$ fatty acid, carries a rather bulky headgroup made up of several sugar rings, some of which, sialic acid residues, can be negatively charged (Fig.1). Gangliosides of the ganglio series are classified according to the structure of the headgroup, the number and position of sugars and of the sialic acid residues [3]. The sugars in the main chain are ordered according to a fixed sequence, as well as the position available for a branched sialic acid residue is fixed, so that each molecule can be obtained from the other by adding or subtracting a given sugar chain. This means that not merely sugar units but also their intramolecular interactions are preserved or destroyed when considering different gangliosides. According to the headgroup features gangliosides show different aggregating properties in water solution. It has been shown that the packing of ganglioside molecules into aggregates is almost purely steric [4], i.e. the contribution of a sugar unit whatever is connected to its spatial hindrance rather than to charge effects due to its dissociation degree. This comes from the large and bulky structure of the headgroup which, on the other side, leads most gangliosides, 


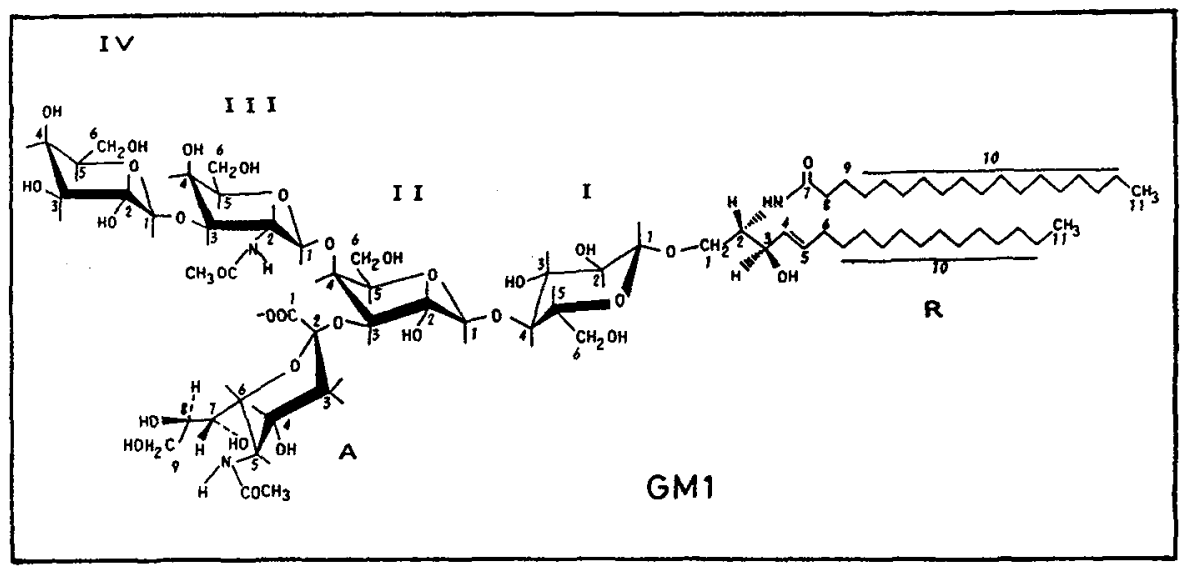

Fig.1. Chemical structure of GM1 ganglioside.

although double tailed, to aggregate in the micellar rather than in the bilayer form. Thanks to the presence of both large hydrophilic and hydrophobic parts in the same molecule, small changes in the monomer headgroup structure sensibly affect the micellar properties. The availability of a considerable number of high purity different gangliosides, prepared by specific chromatographic procedures in order to obtain molecules containing similar lipid moieties, allows significative considerations on the influence of their primary and secondary spatial structure, supporting or stimulating direct observations, e.g. NMR results, of the molecule internal arrangement in the single monomer phase.

\section{GEOMETRICAL PACKING}

The packing of amphiphiles in a micelle obeys some simple rules following the consideration that, except for the surface roughness, no water can exist inside the hydrophobic core, so that one of its dimensions cannot exceed the monomer hydrophobic length, while contact with water is required by the hydrophilic headgroup.

This constraint is very stringent and can be handled theoretically, in the frame of the "opposing forces" model [5]. According to this model, the hydrophobic and surface tension forces are summarized in an attractive interaction acting on the fluid hydrocarbon-water interface; the repulsive forces among headgroups, electrostatic, hydration and steric, are also added up to an equivalent repulsive interaction acting, in the case of small headgroup dimension, on the same interface. At the core surface, then, two opposite forces are applied, the one tending to decrease, the other to increase the interfacial area a per headgroup exposed to the aqueous phase: they balance in correspondence of an "optimal surface area" $a_{0}$, which is assumed to be the area at the interface required by the monomer in the aggregated structure.

Following these observations a simple geometrical sketch for the packing can be drawn [6]. The hydrophobic part of an amphiphile is schematized as occupying inside the aggregate a place with the shape of a truncated cone, identified by three parameters: volume $v$, length 1 , and area $a_{0}$ at the interface. They are summarized in a packing parameter $P=v / a_{0} l$ which assumes the limiting values $1 / 3$ for a true cone and 1 for a cylinder: the corresponding aggregated structures are spheres and bilayers. Micelles are formed when $1 / 3<P<1 / 2$ : in between these values higher $P^{\prime} S$ 


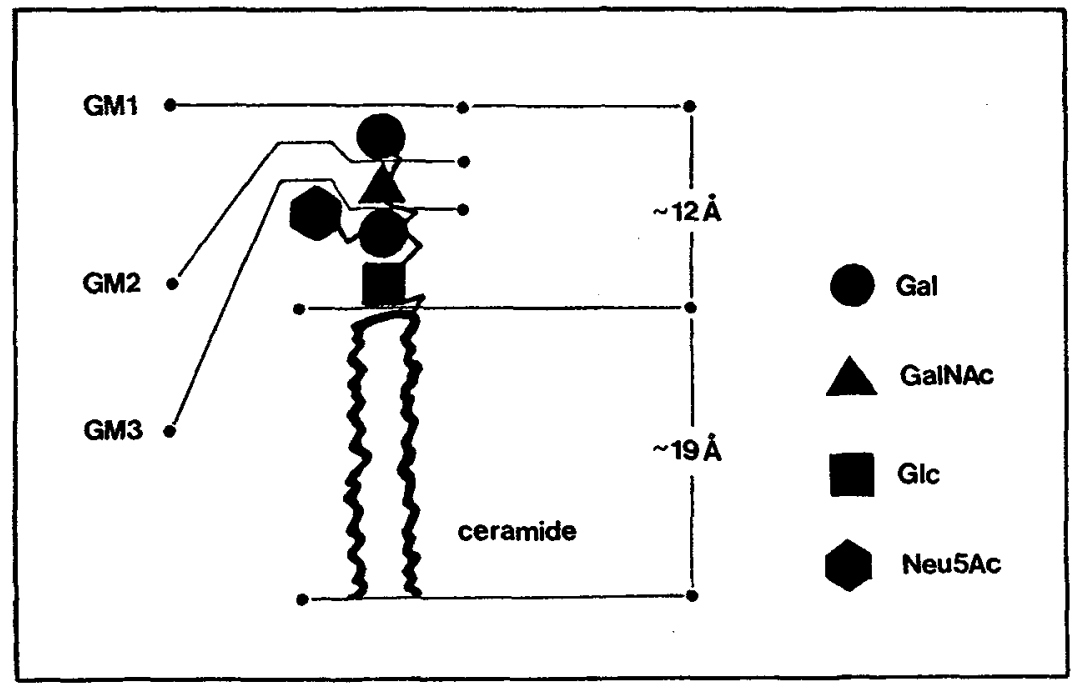

Fig.2. Schematic representation of different monosialo gangliosides.

identify larger and more asymmetrical micelles.

It can be seen that, while $l$ and $v$ can be calculated with good approximation once the chemical structure of the hydrophobic portion of the molecule is known [5], the interfacial area $a_{0}$ is a property of the monomers in the aggregated state, as it is the resulting effect of the complete set of interactions taking place among them. Some additional ambiguity in the evaluation of $a_{0}$ arises when repulsive interactions among lipid chains or among extended and ramificated or charged headgroups have to be taken into account. A detailed schematization of such repulsions being hardly possible, the resulting effect, in the frame of the model followed so far, is that the surface on which the "opposing forces" act can no longer be assumed to be the hydrophilic-hydrophobic one, but some surface displaced a distance $D$ above or below the interface, according to whether tails or headgroups repulsions prevail [7]. Anyway an "optimal surface area at the interface" $a_{0}$ can be defined: it can be calculated a posteriori, once the physical properties of the aggregate are known, or roughly evaluated a priori via some reasonable considerations or by comparison with similar molecules.

We will show that gangliosides, which have all the same hydrophobic portion and display a steric packing of extended and ramificated different headgroups, constitute a particular class in which the geometrical packing properties can be qualitatively attributed to the hydrophilic moiety. In fact different gangliosides will require an interfacial area inside an aggregate as large as to provide in the hydrophilic layer a place wide enough to host the sugar rings and their hydration water. The larger the interfacial area, the smaller the aggregates, with lower aggregation number.

Therefore aggregation properties of gangliosides in solution can be profitably used to reveal small conformational changes in the monomers starting from their cooperative behaviour.

\section{RESULTS AND DISCUSSION}

Static and dynamic light scattering experiments [1] have been performed 
on aqueous solutions of the gangliosides GM1, GM2, GM3, GM4, GD1a, GD1b, GDIb-I, GTIb, GalNAC-GDIa in the millimolar range. A small amount of $\mathrm{NaCl}$, to a final $30 \mathrm{mM}$ ionic strength, has been added to the solutions in order to screen the intermicellar electrostatic repulsion, which does not affect the sterically driven packing behaviour of gangliosides [4]. Data have been treated to deduce the aggregate physical parameters listed in Table 1.

TABLE 1. Physico-chemical parameters of different gangliosides: monomer molecular weight $M W$, number of sugars in the headgroup $S$, aggregation structure and number $N$, interface area per monomer $a_{0}$ and hydrodynamic radius $R_{H}$.

\begin{tabular}{|c|c|c|c|c|c|c|}
\hline & MW & $\mathrm{S}$ & & $\mathrm{N}$ & $\mathrm{a}_{\mathrm{o}}\left(\AA^{2}\right)$ & $\mathrm{R}_{\mathrm{H}}(\AA)$ \\
\hline GM1 & 1560 & 5 & micelle & 301 & 95.4 & 58.7 \\
\hline GM2 & 1398 & 4 & micelle & 529 & 92 & 66 \\
\hline GM3 & 1195 & 3 & vesicle & $\sim 14000$ & -80 & $\sim 250$ \\
\hline GM4 & 1015 & 2 & vesicle & $\sim 18000$ & $\sim 80$ & $\sim 270$ \\
\hline GD1a & 1851 & 6 & micelle & 226 & 98.1 & 58 \\
\hline GD1b & 1851 & 6 & micelle & 170 & 100.8 & 52 \\
\hline GT1b & 2142 & 7 & micelle & 176 & 100.8 & 53.2 \\
\hline GD1b-L & 1851 & 6 & micelle & 229 & 97.6 & 57 \\
\hline GalNAC-GD1a & 2072 & 7 & micelle & 246 & 97 & 60 \\
\hline
\end{tabular}

We consider first the M-series, that is the monosialogangliosides GMI, GM2 and GM3. As shown in Fig.2, the sequence can be obtained by progressively cutting the external sugar in the main chain. The primary effect is that of reducing the hydrophilic character of the amphiphilic molecule. The resulting effect on the aggregative properties can be appreciated by looking at Table 1: an increase of the aggregation number and even a dramatic change from micellar to vesicular structure is observed in the sequence. Those features are expected and can be accounted for by simple considerations. As the number of sugar units decreases, a weaker hydrophilic repulsive contribution to the "opposing forces" balance is given and then a smaller interfacial area is required by the molecule in the aggregate, which becomes less curved and bigger. Therefore the aggregation number increases.

Although showing some differences with respect to other gangliosides, GM4 follows the same trend. GM4 carries a hydrophobic portion slightly more extended than usual and differs from GM3 because it lacks the Glc group. Without going into deeper details, the first order result is that the hydrophilic character of GM4 is surely less marked than that of GM3, which reflects in its aggregative behaviour.

Some more complex considerations can be made by looking at gangliosides belonging to the series GM1, GD1a, GD1b, GD1b-L and GT1b. As shown in Fig.3, these molecules carry the whole 4-sugars neutral main chain and differ in the number and disposition of the branched sialic acid residues, the contribution of which to the packing in the aggregate, as already mentioned, is merely due to their physical dimension.

As far as only the extracted sequence GM1, GDIa and GTIb is concerned the simple number-of-sugars rule is obeyed: more and more hydrophilic 


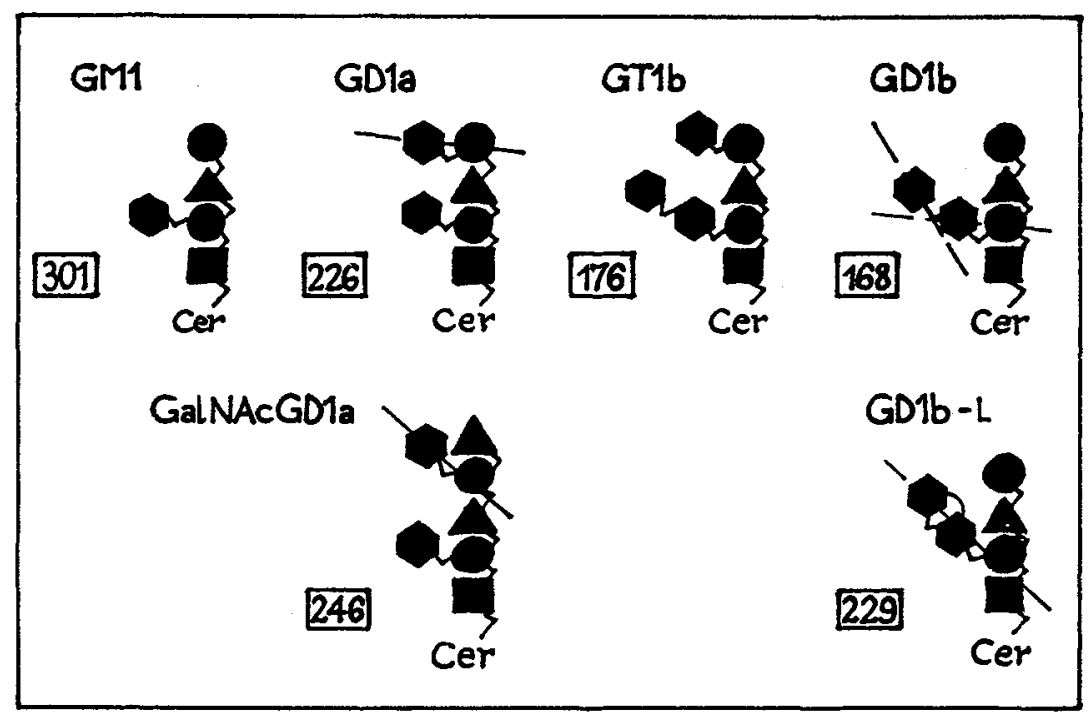

Fig.3. Schematic representation of different gangliosides: the corresponding aggregation numbers are reported in cases.

repulsive contribution is payed by 5,6 and 7 sugar units respectively, resulting in progressively smaller aggregates, with lower aggregation number.

Some confusion arises when considering GDIb and GDIb-I, which stand out of the simpler rules described above. GD1b, which has the same number of sugars, both neutral and dissociating, as GDla, behaves like GT1b, a more hydrophilic ganglioside. GD1b-L, which displays a small chemical modification with respect to its parent GDIb, is similar to GDla. Moreover the more complex ganglioside GalNAC-GD1a, which carries 7 sugar units as GT1b, packs tighter than GDla, which carries only 6 .

This means that a level has been reached at which the extended and ramificated structure of the ganglioside headgroup can no longer be underextimated as in a rough hydrophilicity evaluation. In the frame of the model described above, this is equivalent to notice that the steric repulsion among headgroups plays some additional role, resulting in the fact that different gangliosides displace the "opposing forces" surface at different distances below the hydrophilic-hydrophobic interface.

We propose a geometric specification of this idea and show that it comes out to be useful in the interpretation of the aggregation properties of the complete ganglioside sequence.

The whole ganglioside molecule can be seen to occupy, inside an aggregate, a solid angle which, besides being dictated by the ceramide hydrophobic core general rules, is sensibly affected by the headgroup conformation. This leads to the identification, along the headgroup extension, of "hot" or "cool" environments according to whether significative or negligible changes in the solid angle requirements are brought about by modifications in such positions.

With these considerations in mind, we look first at GD1b. According to the "hydrophilicity" rule, GD1b micelles should have the same aggregation number as GDla, having the same sugars both neutral and dissociating; but the different disposition of the second sialic acid residue plays an important role. The second sialic ring, which in GDla is located in an external position, bound to the last neutral sugar of the main chain, in 
GD1b is branched to the first sialic, closer to the hydrophilichydrophobic interface. More room is then needed by the monomer at this level. This means that a wider solid angle per GDib molecule is required inside the micelle, which reflects in a smaller aggregation number. The relevant influence of the structure of the headgroup in the firstsialic environment is confirmed by comparing the micellar properties of GD1b and GT1b. Although carrying one more sugar, a sialic ring placed at the end of the main chain, and then having a higher hydrophilic character, GT1b forms micelles with the same aggregation number as GD1b. This means that the solid angle required by the two-sialic-group of sugars in the inner position is wide enough to host an additional sialic in an external "cooler" position.

Some deeper considerations can be made by looking at the aggregation properties of GDIb-L as compared with GD1b. GDIb-L, the monolactone derivative of GDIb, shows a chemical modification in the two-sialic group, with the formation of an inner ester. The aggregation number of GDIb-I micelles is higher than GDIb and close to GDla: the increase of the solid angle to locate a second sialic in an inner position is no more needed. By proton NMR, using noe effect, the monomer conformation can be directly evaluated with confidence. The comparison of GDIb and GDIB-L 3Dstructures [8] reveals that the chemical modification induces a spatial rearrangement of the two-sialic group, forcing it to be more lined up with the neutral chain, reducing the angle between the neutral chain and the first-sialic axis. GDIb/GDIb-L case is then a nice example of how ganglioside micellar parameters are sensitive to the secondary structure of the headgroup, and then be used, once a proper model is followed, to support direct observations at the molecular level.

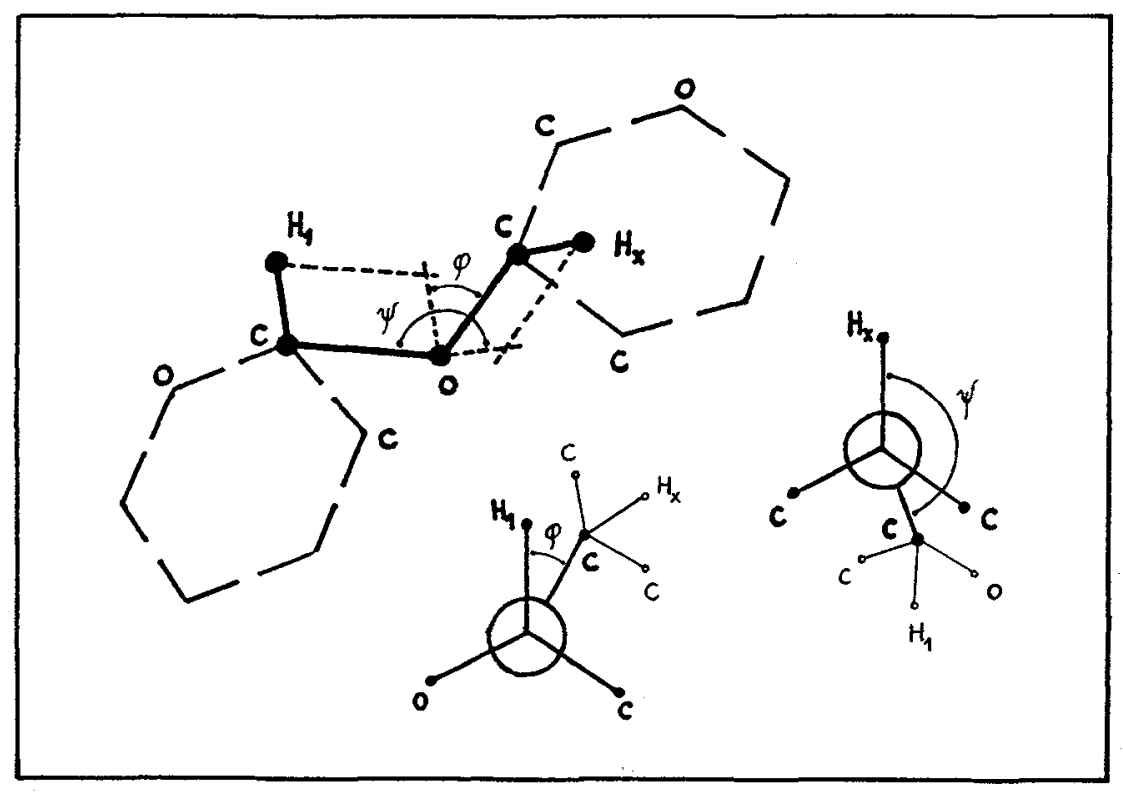

Fig.4. Definition of the $\varphi$ and $\psi$ torsional angles around the bond axes between sugar units according to NMR nomenclature.

A second interesting example is provided by GalNAc-GD1a, although it requires some more bit of information to be appreciated. 


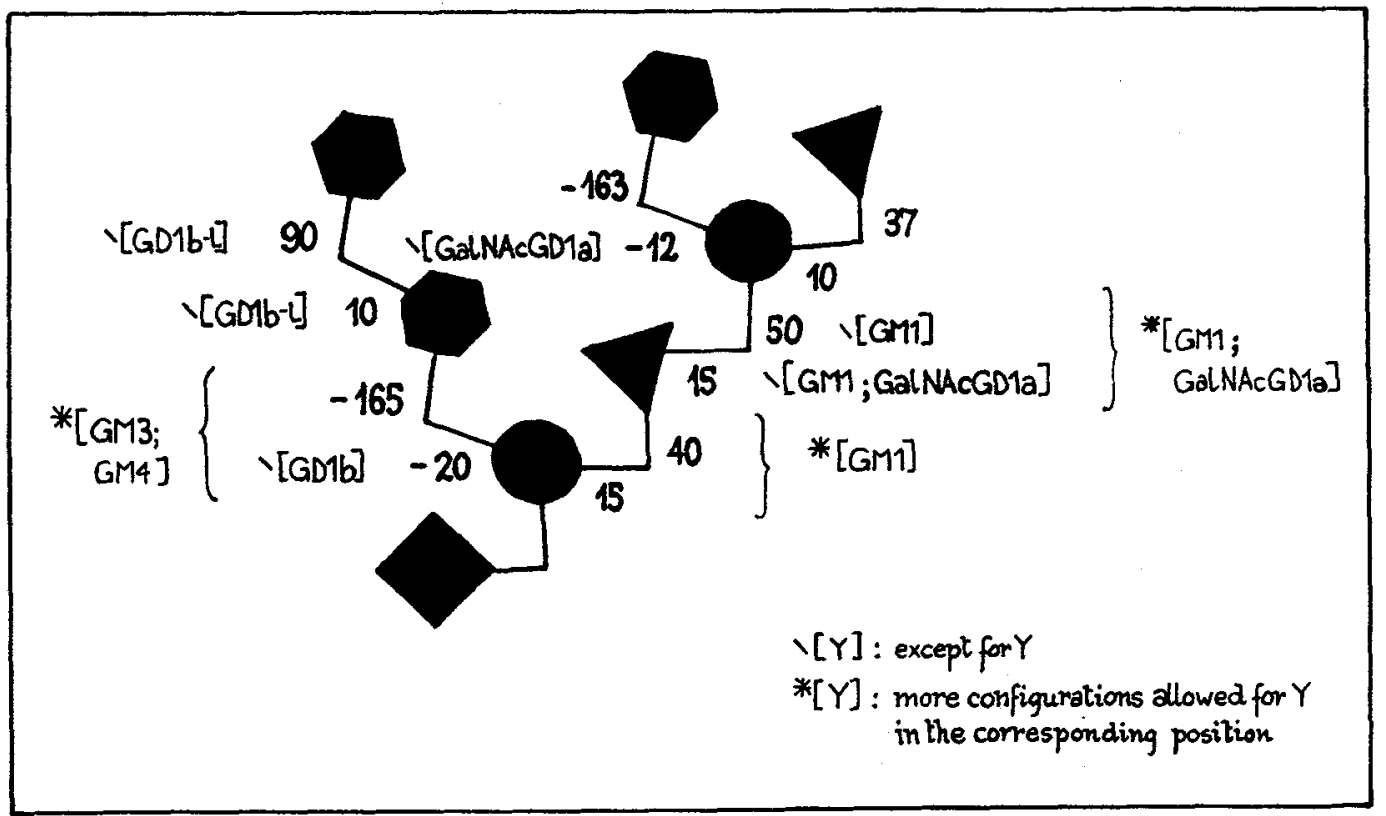

Fig.5. Synoptic representation of the general values assumed by torsional angles in the headgroup of gangliosides: molecules which display configurations different from the general one are specified, as well as molecules in which more than one configuration is allowed $[8,9,10]$.

As already said, the sequence of sugars in a ganglioside is fixed, so that different gangliosides may have or lack not only sugar units but also their complex of interactions. In particular the trio made up by the second and third sugars in the neutral chain and the inner sialic, namely GalNAC-Gal-Neu5AC, is thought to form a strongly interacting bulky superunit. NMR observations on GM3 [9] as compared with GM1 suggest that the presence of GalNAc sensibly limits the rotational degrees of freedom of Neu5AC around its bond axes to Gal, while the lack of GalNAc should destroy the superunit and release Neu5Ac from a tight-to-the-neutralchain position. As a result a ganglioside molecule lacking a GalNAc group is likely to require a wider solid angle in an aggregated structure.

This behaviour has been verified by comparing the aggregative properties of GalNAC-GDla, which carries a second GalNAc-Gal-Neu5Ac trio on top of the first one, to those of the micelle-forming GD1a, which lacks the external GalNAc. Far from following the number-of-sugars rule, the aggregation number of GalNAC-GDla is slightly higher than GDla, as can be explained by considering the different mobility conditions of the external sialic ring.

In addition it is shown that the secondary steric configuration of the environment of the sialic ring, even if located in an external "cool" position, is effective on the packing requirements of the monomer. With this consideration in mind we can comment on GM3 aggregates.

As already said GM3 forms vesicles: the hydrophilic contribution to the "opposing forces" supplied by only three sugars in the headgroup is not enough to support a micellar structure. In the geometrical sketch, this means that the headgroup requires a surface area $a_{0}$ smaller than needed in an aggregate as curved as a micelle, or, equivalently, a solid angle shallow enough to pack into a bilayer structure. 
On the other side, GM3 vesicles have been observed to be interdigitated: in this configuration a larger surface area is available per monomer with respect to the non-interdigitated situation, roughly as twice as large if full interdigitation occurs.

This behaviour is likely to be attributed to the hindrance of the monomer headgroup, bigger than for usual bilayer-forming amphiphiles, which requires a surface area exceeding the lipid chains lateral dimension: considering that GM3 lacks the internal GalNAC, we can imagine that a positive contribution to this feature may be due to the "mobile" conditions of the sialic ring environment.

\section{REFERENCES}

1) M.Corti, V.Degiorgio, R.Ghidoni, s.Sonnino and G.Tettamanti, Chem. Phys.Lipids, 26 (1980) 225;

M.Masserini, s.Sonnino, A.Giuliani, G.Tettamanti, M.Corti, C.Minero and V.Degiorgio, Chem.Phys.Lipids, 37 (1985) 83 ;

L.Cantù, M.Corti, S.Sonnino and G.Tettamanti, Chem.Phys.Lipids, 41 (1986) 315;

M.Corti, L.Cantu, S.Sonnino and G.Tettamanti in: "New Trends in Ganglioside Research: Neurochemical and Neuroregenerative Aspects", R.W.Ledeen, E.L.Hogan, G.Tettamanti, A.J.Yates and R.K.Yu Eds., Fidia Research Series, vol.14, Liviana Press, Padova (1988); L.Cantù, M.Corti and V.Degiorgio, J.Phys.Chem., 94 (1990) 793; L.Cantu, M.Corti, M.Musolino and P.Salina, Europhys.Lett., 13(6) (1990) 561;

M.Corti, L.Cantì and P.Salina, Adv.Coll.Int.Sci., 36 (1991) 153 ; L. Cantu, M.Corti, R.Casellato, D.Acquotti and S.Sonnino, Chem.Phys. Lipids, 60 (1991) 111;

L.Cantu, M.Corti and M.Musolino in: "The structure and Conformation of Amphiphilic Membranes", R.Lipowsky, D.Richter and.K.Kremer Eds., Springer Proceedings in Physics, vol.66, Springer-Verlag, Berlin Heidelberg (1992).

2) G.Tettamanti, S.Sonnino, R.Ghidoni, M.Masserini and B.Venerando in: "Physics of Amphiphiles: Micelles, Vesicles and Microemulsions", V.Degiorgio and M.Corti Eds., North-Holland, Amsterdam (1985).

3) L.Svennerholm, Adv.Exp.Biol.Med., 125 (1980) 11.

4) L.Cantu, M.Corti and V.Degiorgio, Europhys.Lett., 2(9) (1986) 673; V.Degiorgio, L.Cantù, M.Corti, R.Piazza and A.Rennie, Coll.Surf., 38 (1989) 169.

5) C.Tanford, The Hydrophobic Effect, Wiley, NY (1980).

6) J.N.Israelachvili, D.J.Mitchell and B.W.Ninham, J.Chem.Soc.Faraday Trans.II, 72 (1976) 1525.

7) J.N.Israelachvili, S.Marcelja and R.G.Horn, Quarterly Rev.Biophys., 13,2 (1980) 121 .

8) D.Acquotti, G.Fronza, E.Ragg and S.Sonnino, Chem.Phys.Lipids, 59 (1991) 107 .

9) H.C.Siebert, G.Reuter, R.Schauer, C.W.von der Lieth and J.Dabrowsky, Biochemistry, 31 (1992) 6962 .

10) D.Acquotti, L.Poppe, J.Dabrowsky, C.W.von der Lieth, S.Sonnino and G.Tettamanti, J.Am. Chem.Soc., 112 (1990) 7772 ;

S.Sabesan, J.O.Duus, T.Fukunaga, K.Bock and S.Ludvigsen, J.Am.Chem. Soc., 113 (1991) 3236;

L.Poppe and J.Dabrowsky, Biochem.Biophys.Res.Commun., 159

(1989) 618 .

Work partially supported by the Italian Council of Research (CNR), Progetto Finalizzato Chimica Fine II. 